\title{
Neuroplasticity after olfactory training in post-surgical olfactory impaired patients, using functional magnetic resonance imaging. A randomized pilot study
}

\author{
Francisco García-Huidobro ${ }^{1}$, Cristian Montalba ${ }^{2}$, Andres Rosenbaum ${ }^{2}$, David Jofre ${ }^{2}$, \\ Marcelo Andia ${ }^{2}$, Pablo Villanueva ${ }^{2}$, Claudio Callejas ${ }^{3}$, and Claudia Gonzalez ${ }^{2}$ \\ ${ }^{1}$ Pontificia Universidad Católica de Chile \\ ${ }^{2}$ Pontificia Universidad Catolica de Chile \\ ${ }^{3}$ Pontifical Catholic University of Chile
}

July 18, 2021

\begin{abstract}
Abstract Objectives: Treatment for olfactory dysfunction is challenging due to limited therapeutic options. Olfactory training has shown improvement in smell identification, discrimination, and threshold for odor detection. The primary objective is to assess the effect of olfactory training in patients with olfactory dysfunction after pituitary surgery, using functional Magnetic Resonance Imaging (fMRI). Design: This study was designed as a two-arm pilot clinical trial. Setting: Chilean tertiary hospital providing endoscopic pituitary surgery and olfactory evaluation and treatment. Participants: We included two cohorts of subjects: healthy subjects, and patients with post-surgical olfactory dysfunction. Healthy patients were recruited voluntarily at the ENT clinic to assess fMRI testing paradigms and as a control group for the olfactory dysfunction group. Main outcome measures: The main outcome measures were functional activation analysis obtained by fMRI. Olfactory test assessment scores were also obtained during each testing session. Results: 119 patients that underwent an endoscopic transsphenoidal approach were contacted and surveyed for olfactory dysfunction. Twelve patients met inclusion criteria, but six of them declined to participate. Six patients were randomized using computer-generated random numbers. The patients in the trained group showed a significant improvement in the olfactory test results after olfactory training compared to the no-training group $(\mathrm{P}<$ 0.05). The group that underwent olfactory training showed more areas of activation after training than in baseline analysis ( $\mathrm{P}$ $<0.05)$. Conclusion: Patients that underwent olfactory training showed significant improvement in olfactory testing. fMRI results showed more activated areas in the olfactory training group, which could be compatible with neuroplasticity.
\end{abstract}

\begin{abstract}
Objectives: Treatment for olfactory dysfunction is challenging due to limited therapeutic options. Olfactory training has shown improvement in smell identification, discrimination, and threshold for odor detection. The primary objective is to assess the effect of olfactory training in patients with olfactory dysfunction after pituitary surgery, using functional Magnetic Resonance Imaging (fMRI).
\end{abstract}

Design: This study was designed as a two-arm pilot clinical trial.

Setting: Chilean tertiary hospital providing endoscopic pituitary surgery and olfactory evaluation and treatment.

Participants: We included two cohorts of subjects: healthy subjects, and patients with post-surgical olfactory dysfunction. Healthy patients were recruited voluntarily at the ENT clinic to assess fMRI testing paradigms and as a control group for the olfactory dysfunction group. 
Main outcome measures: The main outcome measures were functional activation analysis obtained by fMRI. Olfactory test assessment scores were also obtained during each testing session.

Results: 119 patients that underwent an endoscopic transsphenoidal approach were contacted and surveyed for olfactory dysfunction. Twelve patients met inclusion criteria, but six of them declined to participate. Six patients were randomized using computer-generated random numbers. The patients in the trained group showed a significant improvement in the olfactory test results after olfactory training compared to the notraining group $(\mathrm{P}<0.05)$. The group that underwent olfactory training showed more areas of activation after training than in baseline analysis $(\mathrm{P}<0.05)$.

Conclusion: Patients that underwent olfactory training showed significant improvement in olfactory testing. fMRI results showed more activated areas in the olfactory training group, which could be compatible with neuroplasticity.

Key words : Olfaction disorders, Nasal surgical procedures, Olfactory mucosa, Nasal cavity, Olfactory dysfunction.

\section{Key points}

- All patients in the treatment group improved to a normosmic level, in comparison with the control group, where the 3 patients maintain their anosmic status.

- Six-point difference was observed between the scores of two patients in the treatment group before and after olfactory training.

- fMRI showed an increased amount of activating areas in the treatment group in comparison with the no-treatment group after the intervention.

- After training, nine cluster areas activated in the treatment group, and one big voxel cluster in the cerebellum area.

- All patients showed an activation of the trigeminal related areas.

\section{Introduction}

Post-surgical olfactory dysfunction after an endoscopic endonasal approach has been reported in several series, ranging from $0 \%$ to $10.9 \%(1,2)$. This complication is attributed to surgical resection or excessive instrumentalization of the lateral nasal wall, especially the superior turbinate and the mid-superior part of the middle turbinate, areas that include olfactory epithelium(3).

Even though post-surgical olfactory dysfunction is not an uncommon condition, its treatment remains challenging due to the limited therapeutic options(4,5). Currently, some treatment options have been proposed, like administration of alpha-lipoic acid, theophylline, and corticosteroids. Nonetheless there is no gold standard therapy to treat this condition $(4,6)$.

Recently, olfactory training has shown promising results in improving smell identification, discrimination and threshold for odor detection in patients with postinfectious olfactory dysfunction(5,7-9). Also, a systematic review and meta-analysis published by Pekala et al. 2016, suggests that it may be an effective treatment for olfactory dysfunction due to multiple etiologies(6).

Additionally, it has been reported that olfactory training can induce reorganization in functional connectivity networks in major olfactory areas $(8,10,11)$. Nevertheless, no study has evaluated the effect of olfactory training in patients with post-surgical olfactory dysfunction. The primary objective of this pilot study is to assess the effect of olfactory training in patients with post-surgical olfactory dysfunction using fMRI, while patients undergo an olfactory stimulation protocol. Additionally, as a secondary objective, the effect of olfactory training in olfactory identification was assessed using "Sniffing Sticks Screening Test".

\section{Materials and methods}

This prospective single-center, randomized controlled pilot study was conducted in accordance to the Ethics Committee (Blinded for review) (ID 170728010). 
In this study, we included two cohorts of subjects: healthy subjects, and patients with post-surgical olfactory dysfunction. Healthy patients were recruited voluntarily at the ENT clinic to assess fMRI testing paradigms and as a control group for the olfactory dysfunction group. Three healthy subjects with normal olfactory function participated in the study (two males and one female, with mean age 47 years, range $27-60$ ). All 119 patients that underwent a transsphenoidal pituitary surgical approach were contacted. Forty-six patients that referred a smell impairment with onset after the surgical procedure were invited for further assessment. Twelve patients were enrolled (4 males with mean age 62 years, range $50-75 ; 8$ females with mean age 45 years, range $36-64)$. Only patients with a definitive olfactory dysfunction diagnosis were finally included (determined by Sniffing Sticks Screening Test). Six patients were excluded from the study due to voluntary retirement.

Six patients and three healthy controls completed the study protocol. Healthy subjects completed only the first evaluation session, and all subjects in the patient group performed two evaluation sessions. In the first session, all participants underwent olfactory measurement test in order to diagnose olfactory dysfunction and assess severity. In addition, they were examined by an ENT specialist including a complete physical exam and nasofibroscopy. After clinical evaluation, fMRI measurements were performed using a sniffing paradigm and a trigeminal activation paradigm. Following the first session, the six subjects in the patient group were randomized in two groups of three subjects each using a 1:1 allocation ratio. Randomization sequence was generated by using computer-generated random numbers in the Statistical Package for the Social Sciences (SPSS) version 24.0, and each patient was allocated to an intervention group according to its assigned number. The first group was instructed to perform olfactory training over a 12-week period at home, and the second group received no treatment initially. On the second session, after 12 weeks, all patients were invited to repeat the olfactory testing and fMRI measurements, identical to the first session (S.1).

Olfactory dysfunction was assessed using the "Sniffing Stick Screening Test"(12). The test classifies the patients in three grades of severity depending on their performance, using a numerical 0-12 scale system: normosmia (8-12), hyposmia $(5-7)$ and anosmia $(<5)$. Normative studies for the 12-odor test have been performed in several countries and is currently the only validated olfactory performance test in Chile(1316). The Lawton et al. conversion table was applied to our results to obtain an approximate measure of the identification values of the 16 "Sniffing Stick Identification test"(17).

Olfactory training was performed using four essential oils: Lemon (Limonene $67.08 \%$ and b-pinen 12.52\%), eucalyptus glovus (1-8-cineole $60.00 \%$ ), clove (eugenol $75.49 \%$ and eugenol acetate $13.59 \%$ ) and lavender (linalool $36.53 \%$ and linalyl acetate $32.80 \%$ ). Patients were instructed to expose themselves twice a day to each odor, taking deep sniffs for 30 seconds, and resting 10 seconds between each oil. Additionally, they were instructed to evoke a memory or feeling during the odor exposure that was associated with the essential oil's smell. Patients were contacted monthly to maintain compliance and motivation during the training period, and they confirmed that they performed smell training regularly twice per day. At the end of the 12-week training period, both groups were assessed using an olfactory performance test and a fMRI. After the second evaluation session, the group that did not undergo olfactory training received treatment.

For the scanning sessions, an odor infusion system was attached to a single air-line, with a nasal cannula, in order to perform the sniffing paradigm (S.2). This paradigm consisted of four sniffing blocks, and four normal breathing blocks. Each sniffing block consisted of 20 cycles of 40 seconds duration, and each normal breathing block consisted of 30 cycles of 60 seconds duration. The sniffing block was characterized by an odor intake, eucalyptus essential oil at $5 \mathrm{Lt} / \mathrm{hr}$., through the nasal cannula, to assure brain activation. On the other hand, the normal breathing blocks were characterized by non-odorized compressed air (5Lt/hr.) to guarantee odor cleansing of the nasal cannula during resting state fMRI. Also, a trigeminal activation paradigm was performed to assess the trigeminal system's indemnity. This experiment consisted of five stimulation blocks and five resting blocks, each consisting of 10 cycles of 10-second duration. The stimulation block was characterized by the intake of odorless $\mathrm{CO} 2(50 \%$ at $2,5 \mathrm{Lt} / \mathrm{hr}$.) through the nasal cannula.

To compare olfactory performance scores between each group after each session, a Wilcoxon test and U- 
Mann-Whitney test and the alpha level for all statistical tests was set to $\alpha=0.05$.

All patients were scanned on a 1.5T MRI scanner with an 8-element head-coil. Anatomical reference images were acquired using a T1 weighted 3D Turbo Field Echo sequence. Three hundred slices with a voxel size of $0.9 \times 0.9 \times 0.6 \mathrm{~mm} 3$ with a Field of view (FOV) $230 \times 253 \mathrm{~mm}$. Repetition time (TR)/echo time (TE) of 7.4/3.4 ms with an inversion time delay of $876 \mathrm{~ms}$. fMRI images were acquired using $2 \mathrm{D}$ single-shot, echo-planar imagining (EPI), gradient echo sequence. Twenty-five slices with AC-PC line orientation were acquired with a voxel size of $3.28 \times 3.28 \times 5.0 \mathrm{~mm} 3$ with a FOV of $210 \times 210 \mathrm{~mm}$. TR / TE of $2000 / 45 \mathrm{~ms}$.

fMRI data was preprocessed through realignment, slice timing correction, co-registration to the T1 weighted images and normalization to the Montreal Neurological Institute (MNI) space and smoothing with (8x8x8 mm3), using SPM12, implemented in MATLAB 2018a. In addition, preprocessing steps were performed. STROBE reporting guideline was followed for this study.

\section{Results}

Nine patients were recruited, 3 healthy subjects and 6 patients with post-surgical olfactory dysfunction. The subjects in the olfactory impaired group were randomized, 3 in each group. Demographics of the groups are shown in Table 1 . The only difference between the patients in the intervention $\mathrm{v} / \mathrm{s}$ no-intervention group was that 1 patient in the no-treatment group (33\%) referred a social smoking habit.

\section{Insert Table 1}

\section{Olfactory testing}

Healthy controls were categorized as normosmic, obtaining the maximum scores at olfactory testing, in contrast with the patient group that obtained scores between 1 and 7 , which characterize them as anosmic and hyposmic(18).

The patient group was evaluated initially with the "Sniffing Stick Screening test" observing no significant differences between the treatment and no-treatment groups before training. All patients in the treatment group improved to a normosmic level, in comparison with the control group, where the 3 patients maintain their anosmic status. Comparison of olfactory test after training showed a significant improvement in the treatment group $(\mathrm{P}<0.05)$, as shown in detail in Table 2 . After conversion table application to the screening scores, a six-point difference was observed between the scores of two patients in the treatment group before and after olfactory training (Table 3 ).

\section{Insert Table 2 and 3}

\section{Functional MRI results}

Functional data of the sniffing paradigm was submitted for a first and second level analysis using SPM MATLAB toolbox for both the healthy controls and the patient group. fMRI analysis of healthy controls revealed a significant number of areas that were activated during olfactory stimulation (Table 4). Significant differences in activation areas were determined between the healthy control group and the patient group after performing a two-sample T-test (Fig 3).

\section{Insert Figure 1 and Table 4}

Comparison of functional data between the treatment and no-treatment group showed no significant differences in activation areas before olfactory training, after performing a two-sample T-test. After the intervention, fMRI showed an increased amount of activating areas in the treatment group in comparison with the no-treatment group. Nonetheless, no significant differences were determined. Although no differences were found in the prior analysis, statistical significance was achieved when we compared the activation areas after olfactory training with the activation areas before treatment in the intervention group, using each patient as its own control. Before training, only five cluster areas activated. In contrast, after training, nine cluster areas activated in the treatment group, and one big voxel cluster in the cerebellum area (Table 5). 
All patients showed an activation of the trigeminal related areas, like the precentral gyrus, brainstem, insula, and pars triangularis on their first fMRI. The activation of the areas described beforehand could suggest trigeminal pathway indemnity.

\section{Insert Table 5}

\section{Discussion}

The main aim of this study was to assess the neural activation in patients with olfactory dysfunction after pituitary surgery, before and after olfactory training. We showed that compared to healthy controls, the patient group had fewer activation areas. After 12 weeks of olfactory training in the treatment group, we not only achieved a significant improvement in the olfactory identification test, but also showed an increase in activation areas compared to the no-treatment group.

Plasticity for visual and auditory senses has been widely studied over the past years, with reports of neural reorganization processes after functional $\operatorname{loss}(11)$. For the olfactory sense, recent studies have shown a similar phenomenon, which translates into structural and functional alterations of brain structures(11,19). Although the mechanism that explains plasticity and neurogenesis of the olfactory sense is still unclear, both clinical and basic research has shown that it is a highly plastic system that could be influenced by both bottom-up and top-down processes that could induce continuous neurogenesis(20).

Previous studies have demonstrated that olfactory training can partially restore olfactory function due to multiple etiologies $(6,7,20)$. Our results also show olfactory improvement in smell identification and an increase in activation areas, but specifically in post-surgical etiology patients. The treatment group presented new activation areas after olfactory training, recovering some connections that are also present in healthy subjects, like the orbitofrontal cortex and some areas in the cerebellum. Even though these are not classical olfactory areas, both of them have been reported to be involved in smell perception, especially the cerebellum. Several neuroimaging studies have found odorant-induced activation of the cerebellum, being a significant player in odor recognition and discrimination(19). It is possible to infer that surgical trauma damages olfactory epithelium, causing sensory afferent information loss, which subsequently induces a central olfactory network reorganization. This could be associated with the decreased number of activation areas described by Kollndorfer et al $(8,11)$. The therapeutic effects of olfactory training could be attributed to several factors. One of them could be a bottom-up modulation that consists of a repeated exposure of the patient's olfactory epithelium to different essential oils, hence producing an increase in afferent olfactory inputs. Additionally, when we combine this with a top-down modulation task, like asking the patient to associate the smell with a memory or feeling, we can evoke and activate other brain areas linked to the sense of $\operatorname{smell}(21,22)$. Another factor to have in consideration is the indemnity of the trigeminal pathway. Trigeminal perception is independent from olfactory processing, given that it is due to a specific interaction between chemicals and trigeminal chemoreceptors(23). However, almost all odorants also stimulate the trigeminal system in addition to the olfactory system, at least in higher concentrations(23). Consequently, the olfactory and trigeminal systems interact intimately and work together in the perception of an odorant(23). Additionally, Kollndorfer et al. also suggested the intact trigeminal pathway may trigger olfactory function recovery after olfactory training. In our study, at the initial evaluation, all of our patients showed trigeminal related areas activation, such as the precentral gyrus, brainstem, insula, and pars triangularis, which could suggest pathway indemnity(10).

A limitation of this study is the small sample size of six patients with olfactory dysfunction who completed all measurements. Even though our study had statistically significant findings and raised the possibility that olfactory training could induce neuroplasticity processes and improve olfactory identification, larger scale randomized control trials (RCT) are needed to confirm these findings. Another limitation is the lack of a placebo group, because in our study one of the groups did not receive any intervention. This issue has been reported by other authors, citing that odorless training jars are usually detected by the patients or relatives resulting in intervention abandonment(6). Damm et al. faced this matter by using a high-odor olfactory training group, and a low-odor olfactory training group to better control the placebo effect(7). Nonetheless, in our study the low-odor stimulation could have triggered neural reorganization processes; thus, we used no 
stimulation. On the other hand, the use of "Sniffing Sticks Screening Test" could be considered a limitation, due to the fact that we are only able to assess odor identification with this instrument. To date, this is the only validated olfactory performance test in our country, and several other studies have also assessed olfactory function using the same tool $(13,14,16,24)$. Additionally, Lawton et al. calculated and published a conversion table between the 12 Sniffing screening test and the extended 16 Sniffing identification test(17). After this conversion's application, a six-point difference in the score was observed between the before and after olfactory assessment in the treatment group. This result is particularly important because, according to Gudziol et al., TDI score changes greater than 5,5 points are considered clinically significant(25). Since TDI scores are composed of 3 subtests (identification, discrimination, and threshold), a change in 6 points in one of them will result in a clinically significant change in two of our subjects.

\section{Conclusion}

Our results suggest that olfactory training may be a valid therapeutic option in patients with post-surgical olfactory dysfunction. It also shows that the improvement in an olfactory performance test could be associated with an increase in activation of smell related brain areas and a possible reorganization process in the olfactory network, compatible with neuroplasticity. This pilot study supports the idea that a full scale multicenter $\mathrm{RCT}$ is needed to confirm these initial findings.

\section{Conflict of interest:}

The authors state that they have no conflict of interest.

\section{Bibliography}

1. Magro E, Graillon T, Lassave J, Castinetti F, Boissonneau S, Tabouret E, et al. Complications Related to the Endoscopic Endonasal Transsphenoidal Approach for Nonfunctioning Pituitary Macroadenomas in 300 Consecutive Patients. World Neurosurg. 2016;89:442-53.

2. Cheng Y, Xue F, Wang T-Y, Ji J, Chen W, Wang Z, et al. Analyses and treatments of postoperative nasal complications after endonasal transsphenoidal resection of pituitary neoplasms. Medicine (Baltimore). 2017 Apr;96(15):e6614.

3. Berker M, Hazer DB, Yü cel T, Gürlek A, Cila A, Aldur M, et al. Complications of endoscopic surgery of the pituitary adenomas: Analysis of 570 patients and review of the literature. Pituitary. 2012;15(3):288-300.

4. Kim DH, Kim SW, Hwang SH, Kim BG, Kang JM, Cho JH, et al. Prognosis of Olfactory Dysfunction according to Etiology and Timing of Treatment. Otolaryngol Neck Surg. 2017;156(2):371-7.

5. Sorokowska A, Drechsler E, Karwowski M. Effects of olfactory training: a meta-analysis. Rhinology. 2016;

6. Pekala K, Chandra RK, Turner JH. Efficacy of olfactory training in patients with olfactory loss: A systematic review and meta-analysis. Int Forum Allergy Rhinol. 2016;6(3):299-307.

7. Damm M, Pikart LK, Reimann H, Burkert S, Göktas Ö, Haxel B, et al. Olfactory training is helpful in postinfectious olfactory loss: A randomized, controlled, multicenter study. Laryngoscope. 2014;124(4):826-31.

8. Kollndorfer K, Fischmeister FPS, Kowalczyk K, Hoche E, Mueller CA, Trattnig S, et al. Olfactory training induces changes in regional functional connectivity in patients with long-term smell loss. NeuroImage Clin. 2015;9:401-10.

9. Konstantinidis I, Tsakiropoulou E, Constantinidis J. Long term effects of olfactory training in patients with post-infectious olfactory loss. Rhinology. 2016;54(2):170-5.

10. Iannilli E, Del Gratta C, Gerber JC, Romani GL, Hummel T. Trigeminal activation using chemical, electrical, and mechanical stimuli. Pain. 2008;139(2):376-88.

11. Kollndorfer K, Kowalczyk K, Hoche E, Mueller CA, Pollak M, Trattnig S, et al. Recovery of olfactory function induces neuroplasticity effects in patients with smell loss. Neural Plast. 2014;2014. 
12. Kobal G, Hummel T, Sekinger B, Barz S, Roscher S, Wolf S. "Sniffin" sticks": screening of olfactory performance." Rhinology. 1996 Dec;34(4):222-6.

13. Hummel T, Kobal G, Gudziol H, Mackay-Sim A. Normative data for the "Sniffin" Sticks" including tests of odor identification, odor discrimination, and olfactory thresholds: An upgrade based on a group of more than 3,000 subjects." Eur Arch Oto-Rhino-Laryngology. 2007;264(3):237-43.

14. Katotomichelakis M, Balatsouras D, Tripsianis G, Tsaroucha A, Homsioglou E, Danielides V. Normative values of olfactory function testing using the "Sniffin" Sticks'. Laryngoscope. 2007;117(1):114-20.

15. Sorokowska A, Schriever VA, Gudziol V, Hummel C, Hähner A, Iannilli E, et al. Changes of olfactory abilities in relation to age: odor identification in more than 1400 people aged 4 to 80 years. Eur Arch Oto-Rhino-Laryngology. 2015;272(8):1937-44.

16. Hinz A, Luck T, Riedel-Heller SG, Herzberg PY, Rolffs C, Wirkner K, et al. Olfactory dysfunction: properties of the Sniffin' Sticks Screening 12 test and associations with quality of life. Eur Arch Oto-RhinoLaryngology. 2019;276(2):389-95.

17. Lawton M, Hu MTM, Baig F, Ruffmann C, Barron E, Swallow DMA, et al. Equating scores of the University of Pennsylvania Smell Identification Test and Sniffin' Sticks test in patients with Parkinson's disease. Park Relat Disord. 2016;33:96-101.

18. Hudson L, Silva MC, Núñez JC, Gómez R, Venegas-Francke P. [Evaluation of olfaction in healthy subjects using the Sniffing Sticks battery]. Rev Med Chil. 2012;140(4):442-6.

19. Vedaei F, Ali Oghabian M, Firouznia K, Harirchian MH, Lotfi Y, Fakhri M. The human olfactory system: Cortical brain mapping using fMRI. Iran J Radiol. 2017;14(2).

20. Hummel T, Reden KRJ, Hähner A, Weidenbecher M, Hüttenbrink KB. Effects of olfactory Training in patients with olfactory loss. Laryngoscope. 2009;119(3):496-9.

21. Huart C, Rombaux P, Hummel T. Neural plasticity in developing and adult olfactory pathways - focus on the human olfactory bulb. J Bioenerg Biomembr. 2019;51(1):77-87.

22. Soudry Y, Lemogne C, Malinvaud D, Consoli SM, Bonfils P. Olfactory system and emotion: Common substrates. Eur Ann Otorhinolaryngol Head Neck Dis. 2011;128(1):18-23.

23. Tremblay C, Frasnelli J. Olfactory andtrigeminal systems interact in the periphery. Chem Senses. 2018;43(8):611-6.

24. Sorokowska A, Schriever VA, Gudziol V, Hummel C, Hähner A, Iannilli E, et al. Changes of olfactory abilities in relation to age: odor identification in more than 1400 people aged 4 to 80 years. Eur Arch Oto-Rhino-Laryngology. 2015;272(8):1937-44.

25. Gudziol V, Lötsch J, Hähner A, Zahnert T, Hummel T. Clinical significance of results from olfactory testing. Laryngoscope. 2006;116(10):1858-63.

\section{Figures captions}

Fig. 1. Axial mean anatomical images overlaid with: (A) Activation areas of healthy control subjects, and (B) activation areas of the patient group resulting from a combined group Two sample T-test (Uncorrected p-value, thresholded at 0.05).

S.1 Participant flow diagram.

S. 2 fMRI experiment setting: (A) Sniffing paradigm setting for fMRI experiment. (B) nasal cannula setup.

(C) 1.5T MRI scanner setup.

S. 3 Significant FWE-corrected clusters for olfactory disfunction patients under trigeminal stimulation. 


\begin{tabular}{llll}
\hline Parameters & Training group $(\mathrm{n}=3)$ & $\begin{array}{l}\text { No-training group }(\mathrm{n}= \\
3)\end{array}$ & $\begin{array}{l}\text { Healthy subjects' group } \\
(\mathrm{n}=3)\end{array}$ \\
\hline Age & $55(26-75)$ & $55(37-64)$ & $54(27-60)$ \\
Male (\%) & $1(33)$ & $1(33)$ & $2(67)$ \\
Tobacco (\%) & 0 & $1(33)$ & 0 \\
Time since olfactory & $3(2-5)$ & $3(3-5)$ & 0 \\
dysfunction onset & & \\
(years) & & \\
\hline
\end{tabular}

Table 1: Demographics.

\begin{tabular}{lllll}
\hline & Olfactory test score & Olfactory test score & Olfactory test score & \\
\hline & Before Training & After Training & Median & P-value \\
Healthy control group & Healthy control group & Healthy control group & Healthy control group & Healthy control group \\
Patient 1 & 12 & - & 12 & $0.01^{\text {a }}$ \\
Patient 2 & 12 & - & & \\
Patient 3 & 12 & - & & \\
Patient group & Patient group & Patient group & Patient group & Patient group \\
Treatment group & Treatment group & Treatment group & Treatment group & Treatment group \\
Patient 4 & 3 & 8 & 8 & $0.04^{\text {b }}$ \\
Patient 5 & 5 & 10 & & \\
Patient 6 & 4 & 8 & & No-Treatment group \\
No-Treatment group & No-Treatment group & No-Treatment group & No-Treatment group & No-Treat \\
Patient 7 & 7 & 7 & 2 & \\
Patient 8 & 1 & 1 & & \\
Patient 9 & 5 & 2 & & \\
\hline
\end{tabular}

${ }^{\text {a }}$ U-Mann-Whitney test between healthy control group and patient group before training.

b U-Mann-Whitney test between treatment group and no-treatment group after training.

Table 2: Olfactory test results screening scale: normosmia (8-12), hyposmia (5-7) and anosmia $(<5)$.

\begin{tabular}{|c|c|c|c|c|}
\hline & Olfactory test score & Olfactory test score & Olfactory test score & \\
\hline & Before Training & After Training & Difference $^{c}$ & $\mathrm{P}$-value \\
\hline Healthy control group & Healthy control group & Healthy control group & Healthy control group & Healthy control group \\
\hline Patient 1 & 15 & - & & $0.01^{\mathrm{a}}$ \\
\hline Patient 2 & 15 & - & & \\
\hline Patient 3 & 15 & - & & \\
\hline Patient group & Patient group & Patient group & Patient group & Patient group \\
\hline Treatment group & Treatment group & Treatment group & Treatment group & Treatment group \\
\hline Patient 4 & 4 & 10 & 6 & $0.04^{\mathrm{b}}$ \\
\hline Patient 5 & 6 & 12 & 6 & \\
\hline Patient 6 & 5 & 10 & 5 & \\
\hline No-Treatment group & No-Treatment group & No-Treatment group & No-Treatment group & No-Treatment group \\
\hline Patient 7 & 9 & 9 & 0 & \\
\hline Patient 8 & 2 & 2 & 0 & \\
\hline Patient 9 & 6 & 3 & -3 & \\
\hline
\end{tabular}


${ }^{a}$ U-Mann-Whitney test between healthy control group and patient group before training.

b U-Mann-Whitney test between treatment group and no-treatment group after training.

${ }^{\mathrm{c}}$ Difference between olfactory scores before and after olfactory training.

Table 3: Olfactory test results after conversion from the 12 sniffing score to the 16 sniffing score.

\begin{tabular}{llllll}
\hline & & MNI coodinates & MNI coodinates & MNI coodinates & \\
\hline p-value $^{\text {a }}$ & Cluster Size $^{\mathrm{b}}$ & $\mathrm{X}$ & $\mathrm{Y}$ & $\mathrm{Z}$ & Anatomical Label \\
0.005 & 46 & 26 & 68 & -6 & Inferior Temporal gyrus left \\
0.02 & 26 & -42 & 56 & -10 & Frontal Middle Orbital gyrus \\
0.001 & 78 & -50 & 36 & -14 & Frontal Inferior Orbital gryrus \\
0.000 & 464 & -8 & 52 & -20 & Orbitofrontal cortex \\
0.000 & 9015 & -48 & 6 & -6 & Superior Temporal Gyrus left \\
0.000 & 3366 & 56 & 6 & -10 & Superior Temporal Gyrus right \\
0.000 & 397 & -40 & 44 & 6 & Pars Triangularis left \\
0.000 & 476 & 16 & 4 & -12 & Putamen Right \\
0.000 & 444 & -30 & -66 & -28 & Cerebelum 6 left \\
0.000 & 408 & -4 & -58 & 6 & Posterior Cingulate \\
\hline
\end{tabular}

${ }^{\mathrm{a}} \mathrm{p}<0.05$ FWE-corrected at the cluster level.

b Significantly activated clusters with 10 or more voxels.

Table 4: Significant FWE-corrected clusters for healthy controls.

\begin{tabular}{llll}
\hline Before olfactory training & Before olfactory training & After olfactory training & After olfactory training \\
\hline Anatomical Label & Cluster size & Anatomical Label & Cluster size \\
Left Precentral gyrus & 35 & Left Precentral gyrus & 17 \\
Left Inferior frontal operculum & 72 & Right Inferior frontal operculum & 32 \\
Left Cerebellum & 32 & Left Cerebellum & 37 \\
Left Corpus collosum & 12 & Left Medial frontal gyrus & 14 \\
Left Insula & 28 & Left Medial orbitofrontal cortex & 9 \\
& & Right Cerebellum & 129 \\
& & Left Cingulate gyrus & 40 \\
& Right Fusiform gyrus & 18 \\
& & Left Inferior orbitofrontal cortex & 36 \\
\hline
\end{tabular}

Uncorrected p-value, thresholded at 0.001

Table 5: Significant clusters for patients in the treatment group before and after olfactory training. 

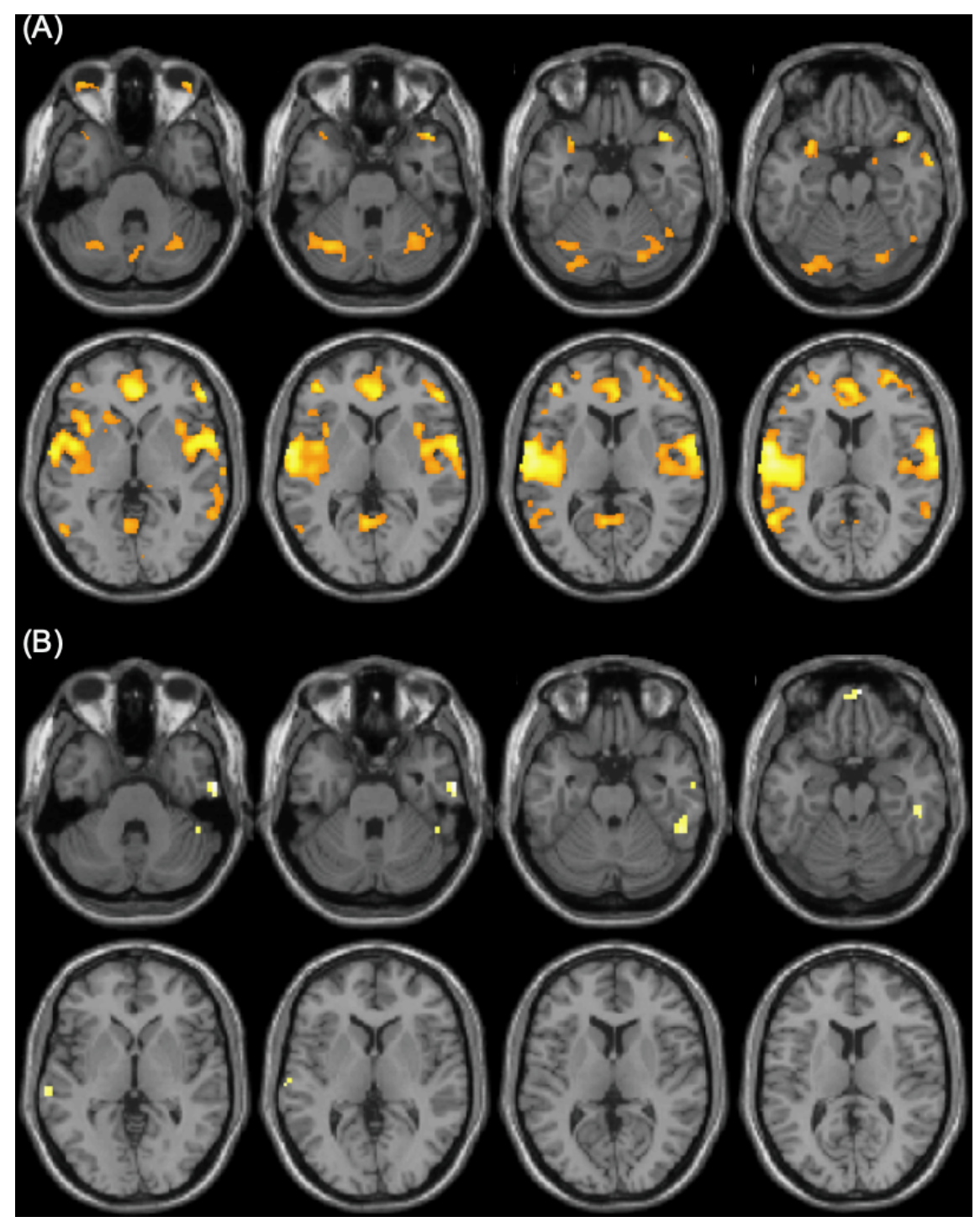